\title{
Electric skyrmions found
}

Nature 568, 368-372 (2019)

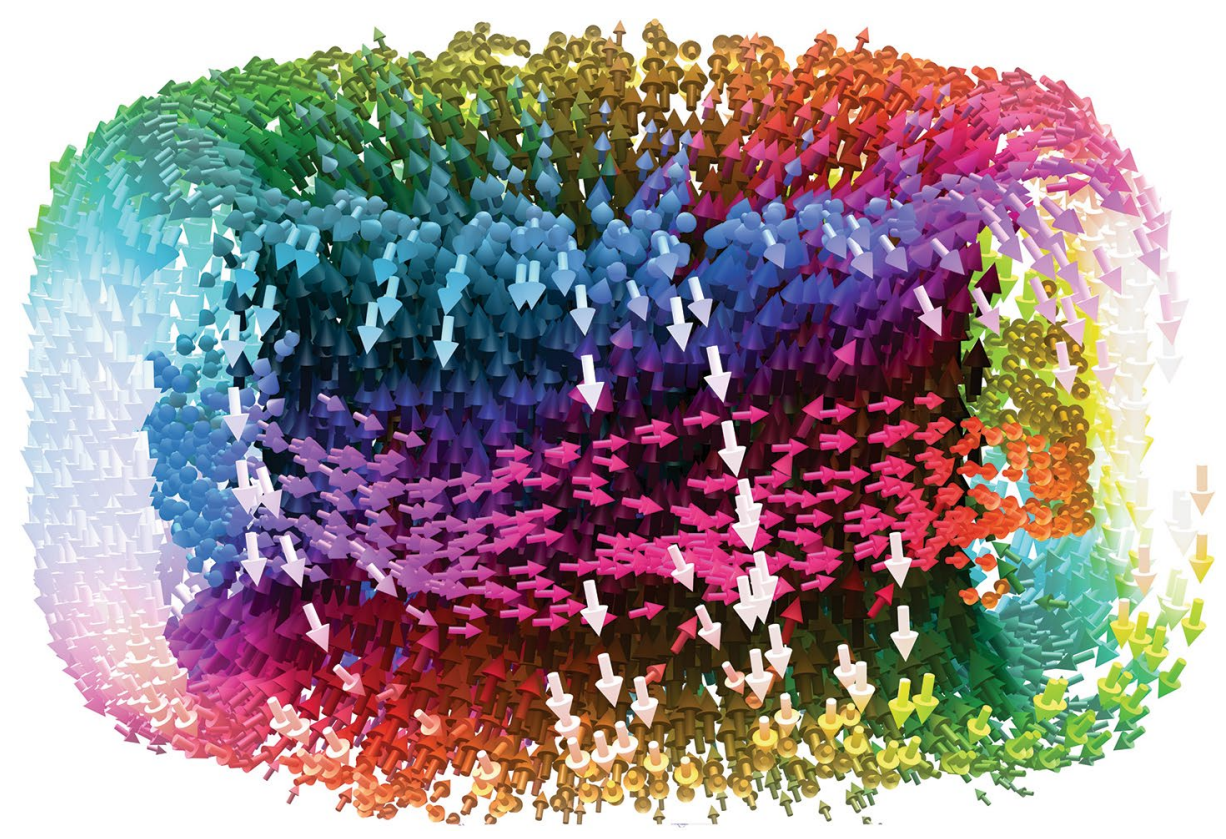

Credit: Xiaoxing Cheng, Pennsylvania State University; C.T. Nelson, Oak Ridge National Laboratory; and Ramamoorthy Ramesh, Berkeley Lab

Magnetic skyrmions are spin textures with nanoscale dimensions, which can be found in magnetic material systems without inversion symmetry. These tiny magnetic whirls can be manipulated by electrical current and could potentially be used as information carriers. Sujit Das, Ramamoorthy Ramesh and colleagues have now observed polar skyrmions in samples of ferroelectric materials that show lattice-mismatch strain.

The researchers - who are based at various institutions in the US, Luxembourg and Spain - examined the arrangement of electric dipoles in superlattice and trilayer structures made of alternating layers of lead titanate and strontium titanate. Electron microscopy imaging of the samples revealed the presence of round features, which were assigned to the formation of room-temperature polar skyrmion bubbles: polarization patterns with regions of opposite electrical polarization. Using $\mathrm{X}$-ray diffraction, Ramesh and colleagues measured circular dichroism in both structures and found evidence of chirality: that is, the overall pattern of dipoles exhibited a left- or right-handed direction of rotation.

Whether these polar skyrmions can be manipulated using electric fields like magnetic skyrmions remains unclear for now.

\section{Christiana Varnava}

Published online: 17 May 2019

https://doi.org/10.1038/s41928-019-0252-z 